\title{
A Phenomenological Finite Element Model of Stereolithography Processing
}

\author{
REOSIVD \\ APR 08 19S6 \\ OSTI
}

\author{
R. S. Chambers, T. R. Guess, T. D. Hinnerichs
}

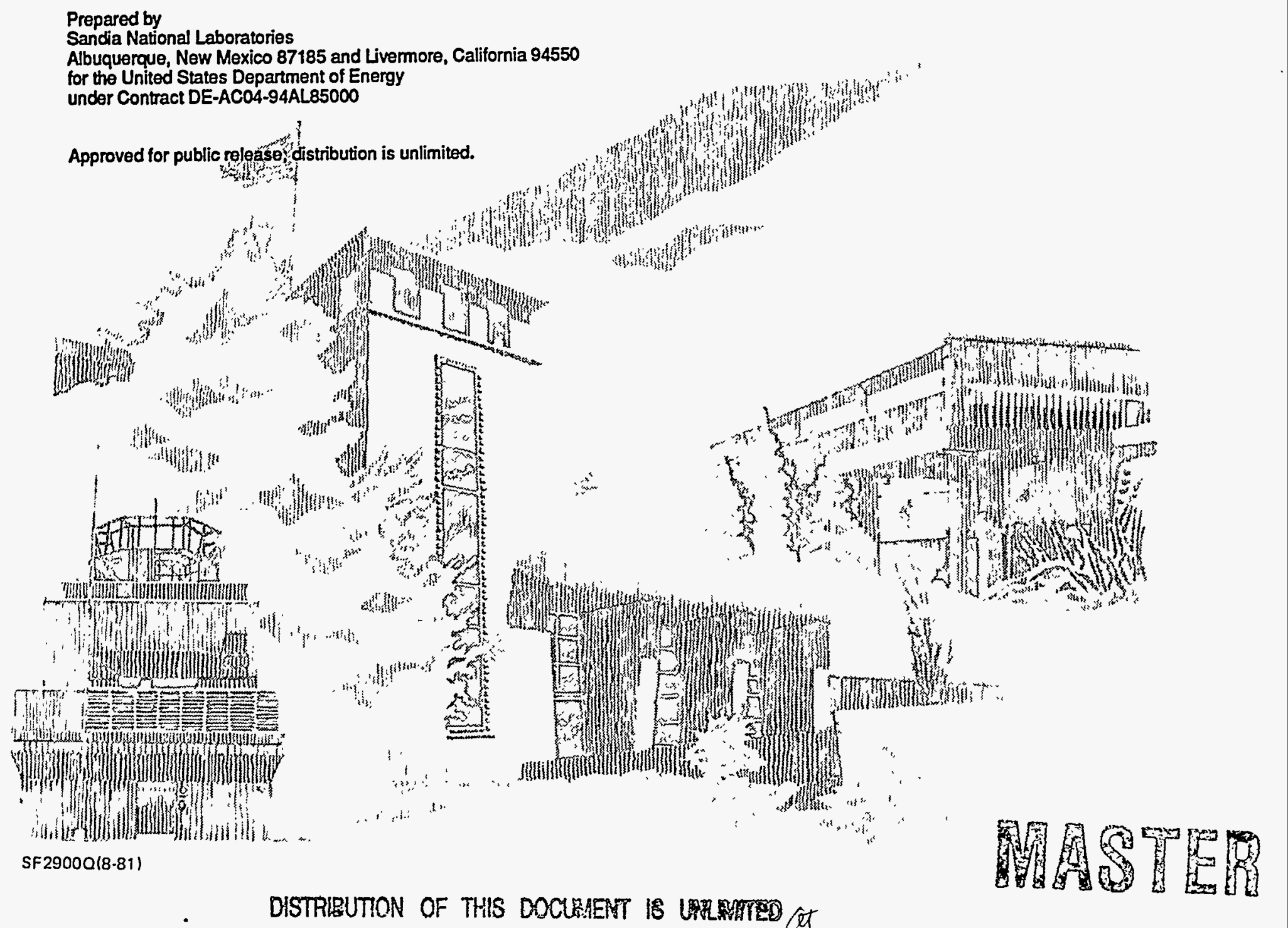


Issued by Sandia National Laboratories, operated for the United States Department of Energy by Sandia Corporation.

NOTICE: This report was prepared as an account of work sponsored by an agency of the United States Government. Neither the United States Government nor any agency thereof, nor any of their employees, nor any of their contractors, subcontractors, or their employees, makes any warranty, express or implied, or assumes any legal liability or responsibility for the accuracy, completeness, or usefulness of any information, apparatus, product, or process disclosed, or represents that its use would not infringe privately owned rights. Reference herein to any specific commercial product, process, or service by trade name, trademark, manufacturer, or otherwise, does not necessarily constitute or imply its endorsement, recommendation, or favoring by the United States Government, any agency thereof or any of their contractors or subcontractors. The views and opinions expressed herein do not necessarily state or reflect those of the United States Government, any agency thereof or any of their contractors.

Printed in the United States of America. This report has been reproduced directly from the best available copy.

Available to DOE and DOE contractors from

Office of Scientific and Technical Information

PO Box 62

Oak Ridge, TN 37831

Prices available from (615) 576-8401, FTS 626-8401

Available to the public from

National Technical Information Service

US Department of Commerce

5285 Port Royal Rd

Springfield, VA 22161

NTIS price codes

Printed copy: A03

Microfiche copy: A01 


\title{
A Phenomenological Finite Element Model of Stereolithography Processing
}

\author{
R. S. Chambers, T. R. Guess, and T. D. Hinnerichs \\ Engineering Sciences Center \\ Sandia National Laboratories \\ Albuquerque, New Mexico 87185
}

\begin{abstract}
In the stereolithography process, three dimensional parts are built layer by layer using a laser to selectively cure slices of a photocurable resin, one on top of another. As the laser spot passes over the surface of the resin, the ensuing chemical reaction causes the resin to shrink and stiffen during solidification. When laser paths cross or when new layers are cured on top of existing layers, residual stresses are generated as the cure shrinkage of the freshly gelled resin is constrained by the adjoining previously-cured material. These internal stresses can cause curling in the compliant material.

A capability for performing finite element analyses of the stereolithography process has been developed. Although no attempt has been made to incorporate all the physics of the process, a numerical platform suitable for such development has been established. A methodology and code architecture have been structured to allow finite elements to be birthed (activated) according to a prescribed order mimicking the procedure by which a laser is used to cure and build-up surface layers of resin to construct a three dimensional geometry. In its present form, the finite element code incorporates a simple phenomenological viscoelastic material model of solidification that is based on the shrinkage and relaxation observed following isolated, uncoupled laser exposures. The phenomenological material model has been used to analyze the curl in a simple cantilever beam and to make qualitative distinctions between two contrived build styles.
\end{abstract}




\section{Acknowledgments}

We would like to thank G. D. McCarty, B. T. Pardo, and D. E. Reckaway for their many hours of work in running the SLA-250 and C. L. Atwood who patiently allowed us to tie-up the SLA-250 for extended periods. We also would like to acknowledge the contributions of M. E. Stavig and L. D. Harwell in instrumentation, P. G. Stomberg in mechanical design, and R. N. Shagam for his cure shrinkage measurements. It is also fitting to express our appreciation to R. P. Chartoff, J. S. Ullett, and A. J. Lightman at the University of Dayton for their assistance in making property measurements and for their SLA software which allowed us to create our own build styles. Manfred Hofman at Ciba-Geigy and Paul Jacobs at 3D Systems were helpful in discussing issues and reviewing the technology. D. B. Adolf and W. R. Witkowski graciously agreed to review this report. 


\section{Contents}

1 Introduction 11

2 Finite Element Methodology 13

3 Phenomenological Material Modeling 16

3.1 Cure Shrinkage Measurements 16

3.2 Stress Relaxation Measurements 18

3.3 Solidification Modeling $\quad 20$

3.4 Modeling Problems and Limitations 20

4 Demonstration Problems 23

4.1 Birthing Elastic Elements 23

4.2 Viscoelastic Analysis of Contrived Build Styles 25

5 Conclusions 32

6 References 33 


\section{Figures}

Figure 1. Schematic of Stereolithography Process

Figure 2. Finite Element Mesh of a Six-Layer Block Used to Model a Simple Cantilever Beam

Figure 3. Schematic Illustrating Depth of Cure and Off-Line Distance Parameters Associated with a Laser Hit Occurring on Finite Element Mesh Layer

Figure 4. Cure Shrinkage Strain Measured in SL 5149 Acrylate Resin After Single Laser Exposure

Figure 5. Cure Shrinkage Strain Measured in SL 5149 Acrylate Resin by the University of Dayton After Second Laser Exposure

Figure 6. - Force Measured in a Single Strand of SL 5149 Acrylate Resin With Constrained Ends Following a Second Laser Exposure

Figure 7. Stress Relaxation Measured in Single Strands of SL 5149 Acrylate Resin Following Multiple Laser Hits (Stress in psi and Time in seconds)

Figure 8. Prony Series Fits of Tensile Stress Relaxation Moduli for Single Strands of SL 5149 Acrylate Resin Following Multiple Laser Hits (Stress in psi and Time in seconds)

Figure 9. Geometry of Cantilever Beam Analyzed During Process Build

Figure 10. Schematics Illustrating the Vector Paths and Drawing Sequence for the Two Build Styles Used in the Analysis of the Cantilever Beam

Figure 11. Plots of the Deformed Mesh Obtained from the Analysis of the Cantilever Beam Built According to the Scheme I Laser History

Figure 12. Plots of Z-Displacement Along the Bottom Face of the Cantilever Beam Following the Solidification of 4 Layers Drawn Using Scheme I

Figure 13. Plots of Z-Displacement Along the Bottom Face of the Cantilever Beam Following the Solidification of 4 Layers Drawn Using Scheme II

Figure 14. Cantilever Beam Geometry Used in Artifact Build and Modeling for Demonstration Problem 2

Figure 15. Transverse Vectors Used to Illustrate Scheme II Build Scheme 28

Figure 16. Dayton 2nd Laser Exposure Data Compared to Model Approximation

Figure 17. Comparison Between the Finite Element Curl Predictions Obtained by Modeling the Scheme I and Scheme II Build Styles in Cantilever Beam

Figure 18. Curl Measured and Predicted in Cantilever Beams Built in SLA-250 According to the Scheme I and Scheme II Build Styles 


\section{Introduction}

The stereolithography process is a rapid prototyping process in which three-dimensional (3D) parts are built layer by layer using a laser to selectively cure sheets of photosensitive resin, one on top of another [1]. The geometric definition of the layers is obtained by constructing a series of slices through a 3D solid model of a part. These slices, when reconstructed in sequence, reproduce the full part geometry. Working from a slice definition file, the stereolithography apparatus uses a focused ultraviolet (UV) laser beam directed by a computer-controlled X-Y scanning mirror system to accurately trace the layer geometry on the surface of the resin. As the laser spot passes over the surface of the resin, the ensuing chemical reaction causes the resin to shrink and stiffen during solidification. A schematic diagram of the process can be seen in Figure 1. As with any rapid-prototyping method where solidifying

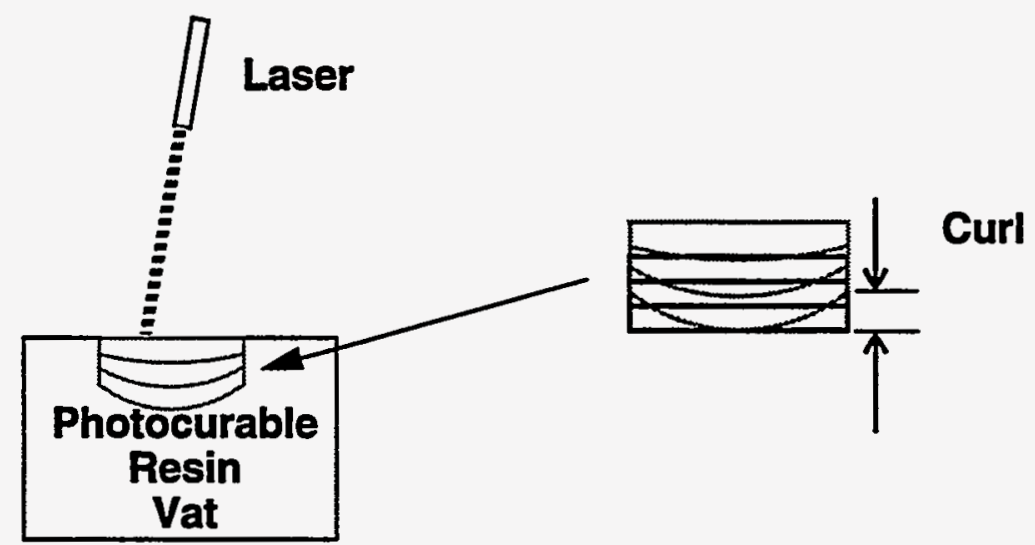

Figure 1. Schematic of Stereolithography Process

materials undergo shrinkage, dimensional tolerances can be difficult to maintain. When laser paths within a layer cross or when new layers are cured on top of existing layers, residual stresses are generated as the cure shrinkage of the freshly gelled resin is resisted by the stiffness of the adjoining previously-cured material. These internal stresses, though small, can cause significant deformations (curling) in the compliant material. The presence of such deformations can lead to distorted parts that do not comply with specified geometric tolerances.

The problem of curl distortion has been recognized and discussed by many authors [2-5]. In fact, standard builds have been used as diagnostics to evaluate the severity of curl $[5,6]$. The curl distortion and warpage characteristic of a layered build are, to a large extent, governed by the temporal and spatial history of solidification directed by the surface tracings of the laser. This recognition has lead to the development of hatch styles, weaves and new materials that reduce part distortions.

Finite element methods are particularly well suited to the task of developing predictive tools for studying highly complex, nonlinear processes. In the current research project, a study of the stereolithography build process was undertaken with two specific objectives being proposed: 
1. to develop the finite element code architecture needed to numerically simulate partbuilding in the stereolithography process, and

2. to investigate the possibility of analyzing part-building using a simple phenomenological model of solidification.

In meeting the first objective, the decision was made to build the code from existing finite element software (i.e., JAS3D [7]) by adding the capability of modeling the historical record of layering and laser tracking. The second objective entailed the highest risk, by far. Here, an attempt was made to characterize the gross behavior of individually solidified strands created by a single pass of the laser and then to use the "average" mechanical properties of the strands in a built-up assembly to model the deformation and stiffness of the total structure. This report provides an overview of the finite element approach and material modeling effort and presents some simple demonstration problems. A detailed description of the material tests and data is available in a separate Sandia Report [8]. 


\section{Finite Element Methodology}

In designing a code architecture for modeling the stereolithography build process, six essential features were identified. These include the ability to:

1. model a layered structure,

2. track the path history within each layer (i.e., laser position and time),

3. connect and join strands and layers following laser curing,

4. model depth of cure effects,

5. depict the parabolic shape of the cross-section of a strand, and

6. account for nonlinear deformations fields (e.g., in-plane and curl).

All of these features are attainable within the framework of the finite element method. Because the finite element method is based on a spatial discretization, models naturally consist of an assembly of elements. Joining and connectivity are directly imposed through the nodal linkage of adjacent elements, and modeling accuracy is achieved by refining the mesh. The orthogonality of hexahedral elements makes them particularly well suited for modeling layered structures.

Because polymerization is initiated by the laser exposure on the surface of the resin, the order of the build process is controlled by the programmed path of the laser. It is the sequence and history of solidification and shrinkage that drives the internal stress state and subsequent curl within layers. To be an effective tool, a numerical model must be capable of distinguishing between path histories. Because the liquid resin, being a low viscosity liquid, quickly flows to relieve stresses, it is the constrained shrinkage of the solidified (i.e., gelled) material that generates residual stresses. Within the finite element context, the stiffness of the material and the path dependence of the process can be achieved by starting with a block of inactive finite elements that are sequentially "birthed" (i.e., activated) according to the prescribed path and speed of the laser draw. Under this scheme, the finite element mesh is constructed to encompass the perimeter of the total volume of liquid resin from which the solid part is to be made. This includes all build layers meshed and assembled into one chunk of material. However, it need not include any resin beyond the external boundaries of the solid part because the liquid resin is assumed to be stress-free and have no mechanical stiffness. Figure 2 contains a

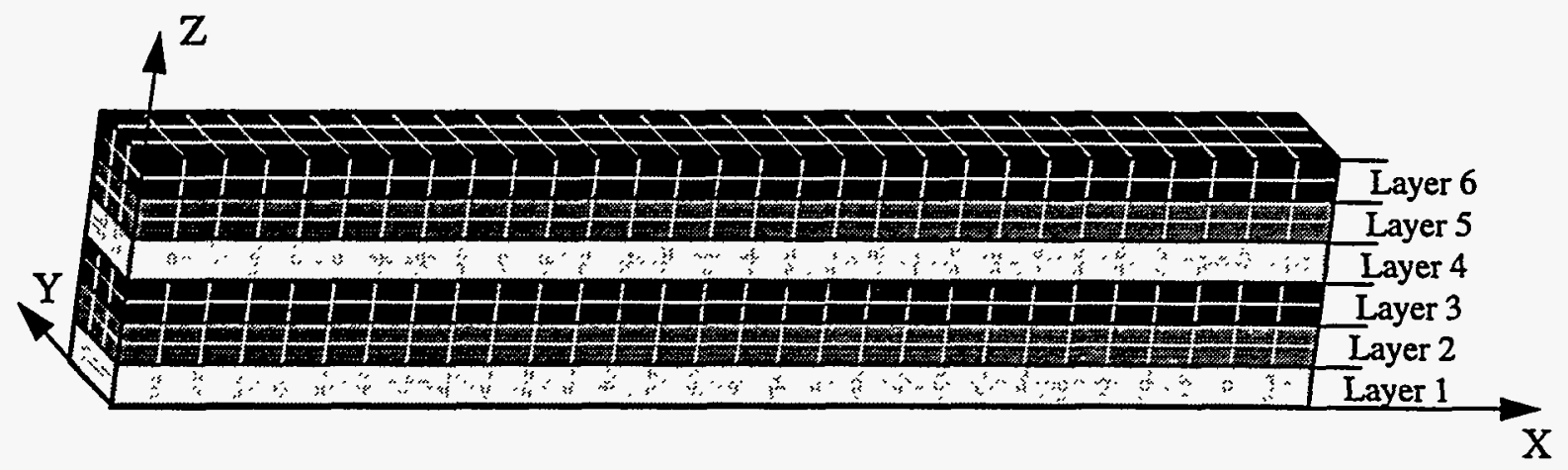

Figure 2. Finite Element Mesh of a Six-Layer Block Used to Model a Simple Cantilever Beam 
finite element mesh of a six-layer block used to model the building of a simple cantilever beam. In this mesh, each of the six build layers was assumed to be 0.010 inches thick, and each layer was subdivided into two planes of elements. A layer (in the context of this description) refers to the thickness of liquid resin that is used by the stereolithography apparatus to construct built-up parts. It is the thickness of liquid resin deposited on previously cured layers by lowering and raising the elevator and squeegeeing the surface in preparation for subsequent laser processing. By convention, a right-hand coordinate system is used to orient the mesh so that the fluid surface (i.e., top surface of an undeformed layer) is an X-Y plane, and the built-up layers are added in the positive $\mathrm{Z}$ direction. Using this convention, layers can be identified by the Z-coordinate of their top surface which corresponds to the resin fluid level at the time that layer is processed.

At the start of an analysis, all elements are assumed to be inactive reflecting the initial stress-free condition of the liquid resin. The serial nature of the part build is represented by birthing (i.e., activating) finite elements along the predetermined coordinate paths traced by the laser in each individual layer as it is being processed. During the course of an analysis, layers are processed in sequence starting from the bottom of the mesh block and proceeding to the top following the pattern of what occurs in the stereolithography apparatus. The laser path vectors are stored in an ASCII file and read by the finite element code in a fashion completely analogous to the method whereby the stereolithography apparatus reads a slice file to define the part build. The first record in the ASCII path file contains laser process parameters including such things as: 1) laser spot radius, 2) laser "on" drawing speed and depth of cure, 3) laser "off" positioning speed, 4) time delay between processing adjoining layers (i.e., time required to lower/raise elevator, squeegee, etc.), and 5) some mesh parameters defining the layer thickness and number of elements in the fluid plane. Subsequent records in the path file contain data sets, one per layer, with each data set defining the vector paths for one layer in the mesh block. The first record in each layer's data set contains a "LAYER" identifier along with the Z-coordinate uniquely identifying this layer by the location of the top surface of the layer. The remaining records in the data set contain pairs of $X-Y$ coordinates associated with the beginning and end points of each "straight-line" vector that is to be drawn. Within the finite element program, there is logic designed to scan the finite elements at the surface of the current layer (i.e., the layer currently being processed) and determine when and if these elements fall within the width of the radius of the moving laser spot. If so, then the elements are perceived to be hit and activated when the spot center passes by the centroid of each element. The time of activation is computed by summing the drawing, positioning, and delay times up to the time of the hit. As such, the actual processing time is tracked and computed through the specified vector history. At the time of activation, the elements are brought into existence and begin solidifying and shrinking according to the code's time-dependent phenomenological model of curing. Structural linkage is enforced naturally [9] by activating elements to join strands or layers when laser paths connect or overlap, respectively.

By default, for each finite element in the mesh, the code tracks a number of process parameters believed to be important to the material response. This includes the number of times that an element has been hit by the laser and the time of each hit. Furthermore, as each hit occurs, the code computes the depth, D, of the element's centroid below the surface and its perpendicular, off-line distance, S, from the Z-plane of the laser path, based on the current 

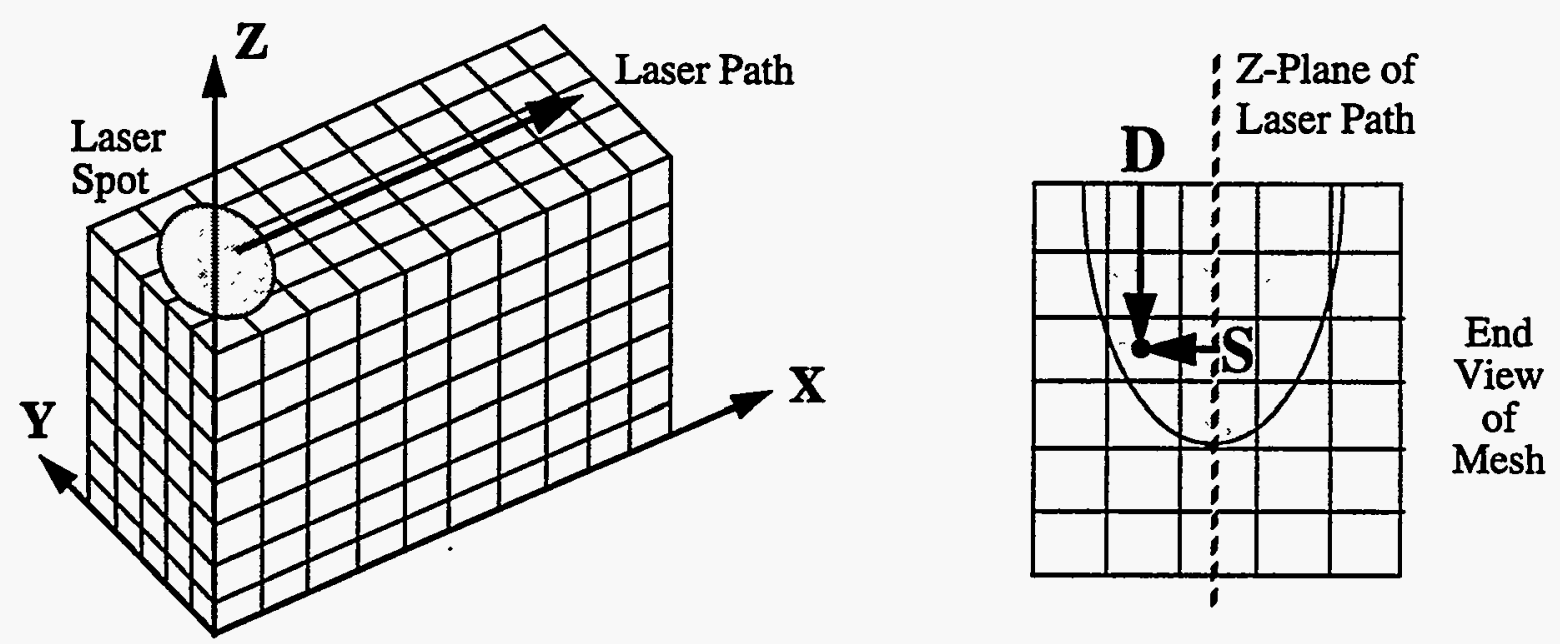

Figure 3. Schematic Illustrating Depth of Cure and Off-Line Distance Parameters Associated with a Laser Hit Occurring on Finite Element Mesh Layer

model coordinates. Figure 3 contains a schematic defining these parameters. The computations are ultimately important for determining depth of cure and exposure in underlying elements. With this information, it is possible to activate a parabolic cross-section of finite elements extruded in space along the line of laser drawing consistent with the predictions [5] for an actinic Gaussian laser beam moving at constant velocity across a photopolymer obeying the Beer-Lambert Law. In Figure 3, the laser spot and the parabolic cross-section of laser exposure below the surface of the resin are shaded grey. The approximation of the parabolic cross-section can be represented by activating those finite elements whose centroids fall within the shaded domain. Although the rectangular shapes of the activated finite elements render a jagged boundary, the boundary can be smoothed by further mesh refinement. In the present version of the code and the demonstration problems subsequently considered, the strand is approximated by a rectangular cross-section. 


\section{Phenomenological Material Modeling}

The stereolithography process encompasses a broad range of extremely complex material behavior. From the physics of the UV laser exposure through the chemical kinetics of an exothermic reacting system to the solidification, cure-shrinkage, and viscoelastic relaxation and aging of the gelled solid. In the current program, no attempt has been made to accurately account for all such behavior. Rather, a computational framework has been developed to model a few features of the build process with the idea that more complex behavior can be incorporated into the model as opportunities and needs arise.

It is well known that a strand of resin cured by a single pass of the laser exhibits a gradient in the extent of reaction through the depth of cure which likewise induces a gradient in cure-shrinkage and mechanical properties as well. Moreover, as the material is subjected to multiple hits from the laser, the exposure increases and the depth of cure is enhanced. The second objective of this project was to determine whether a simple phenomenological engineering model of solidification can be used to make qualitative distinctions between build styles. The current version of the code, lacking a complete kinetic theory with coupled viscoelastic material model, assumes that the strands cured during a laser pass are homogeneous. This phenomenological model, thus, uses an "average" set of properties for the composite section of the strand and is consistent with the manner in which experimental properties have been collected in-situ for individual strands of resin [8, 10-12]. In these tests, cure shrinkage and stress relaxation data have been measured on strands of resins subjected to multiple laser hits. The tests were used to characterize the depth of cure and "average" material properties as a function of the "number of laser hits".

Tests were conducted on two different Ciba Geigy resins: 1) Cibatool SL 5170 epoxy and 2) Cibatool SL 5149 acrylate ester. Both materials are one-component, photocurable liquid resins designed for $\mathrm{HeCd}$ laser-based stereolithography equipment, especially for the 3D Systems SLA-250 [1]. Data were collected from tests at Sandia and from tests conducted under contract with the University of Dayton [13]. The results are documented in a separate report [8]. In this section, some of the phenomenological material properties are summarized for the acrylate resin to facilitate a discussion of model development. The acrylate was selected as the primary modelling candidate because acrylate parts exhibit greater amounts of curl than the epoxy parts.

\subsection{Cure Shrinkage Measurements}

The cure-shrinkage strains were measured in two different ways. The Sandia data were collected using a non-contact video method $[8,10,11]$ in which single resin strands were fixed at one end while the contraction of the free end was measured as a function of time. A typical shrinkage strain measured following a single laser hit is shown in Figure 4. The data show the total linear cure shrinkage strain of the gelled resin to be about $1 \%$ recorded over a period of 3 minutes. Because the tip of the sample tends to sink after about 5 minutes, this method could not be used to measure the cure shrinkage associated with a second laser hit.

The shrinkage method employed at the University of Dayton was an elaborate contact method [12] which used a lever arm attached to a micro-balance mechanism to deflect a light beam as the polymer shrinks. This technique was able to measure shrinkage following multi- 


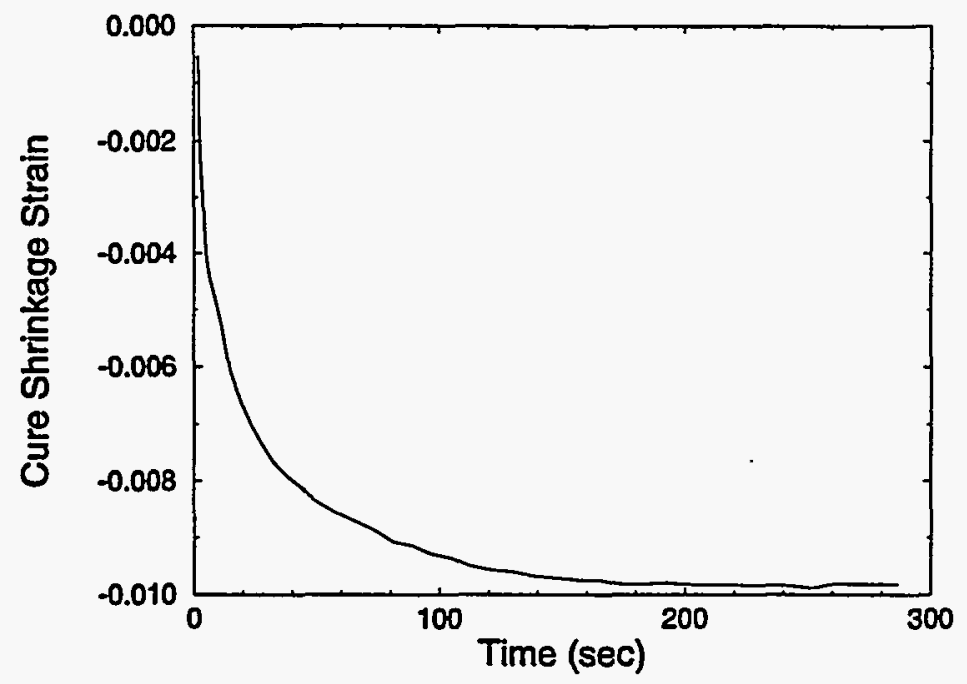

Figure 4. Cure Shrinkage Strain Measured in SL 5149 Acrylate Resin After Single Laser Exposure

ple laser hits. Although the sample sizes and laser exposures adopted for the Dayton tests differed from the parameters employed in the Sandia experiments, the shrinkage strain measured after a single laser hit was comparable to the result illustrated in Figure 4. There was, however, one significant difference. The Dayton measurements detected swelling in the later stages of the test. The effect of this behavior was to reduce the total cure shrinkage somewhat and to replace the asymptotically flat appearance of the data in Figure 4 with an up-turn in the curve after about two minutes. The cure shrinkage measured at the University of Dayton following a second laser exposure, 5 minutes after the first exposure, is shown in Figure 5 . In this plot, the shrinkage has been zeroed at the time of the second laser hit. The magnitude of the cure shrinkage measured as a result of the second laser hit (i.e., $0.8 \%$ ) was less than the cure shrinkage measured following the first hit. This trend was observed in repeated laser strikes. In instances where multiple laser strikes occurred over short time intervals where the reaction

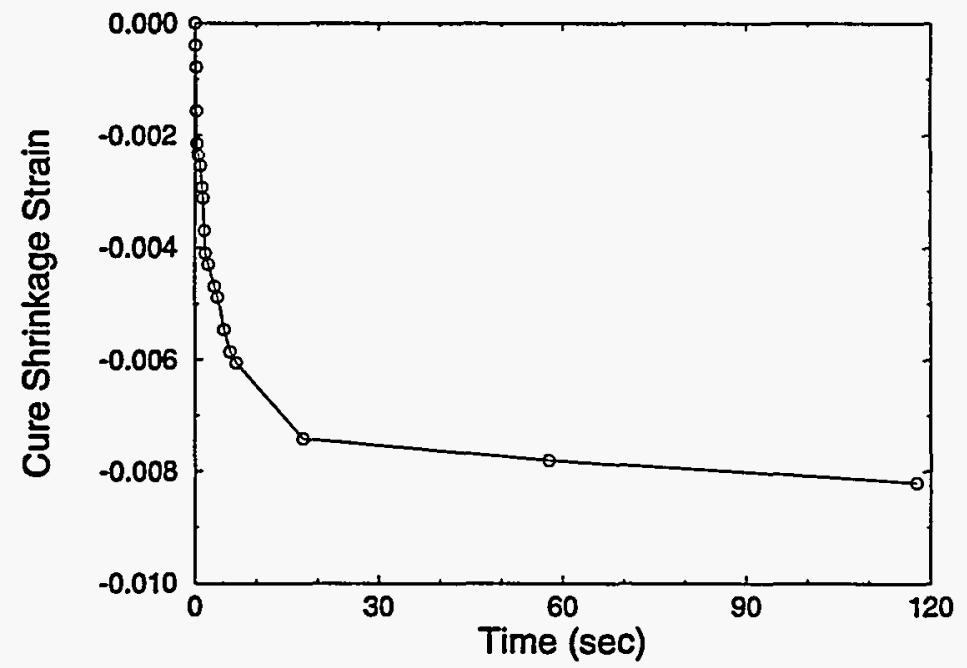

Figure 5. Cure Shrinkage Strain Measured in SL 5149 Acrylate Resin by the University of Dayton After Second Laser Exposure 
from one exposure was not allowed to terminate before the next exposure, the observed shrinkage was different. Hence, shrinkage was clearly observed to be not only a function of the number of laser hits but also the time of exposure relative to prior history.

\subsection{Stress Relaxation Measurements}

Stress relaxation tests on individual strands of resin were conducted by drawing strands with one end attached to a load cell while the other was attached to the end of an electronically driven micrometer $[8,10,11]$. In this configuration, the micrometer can be programmed to impose a "step-like" strain, and the load cell can measure the accompanying force history. Although the original intent was also to measure the force generated by cure shrinkage following the very first laser exposure as the strand was connected to the load cell and micrometer head, the resolution of the load cell ( 0.02 grams) was not sensitive enough for this purpose. No detectable force was measured during this gelation and first shrinkage of the material. There was, however, sufficient resolution to measure a "cure-shrinkage" force generated following subsequent laser hits in this constrained configuration (i.e., while the ends were attached to the load cell and micrometer head). Figure 6 contains a plot of the force measured

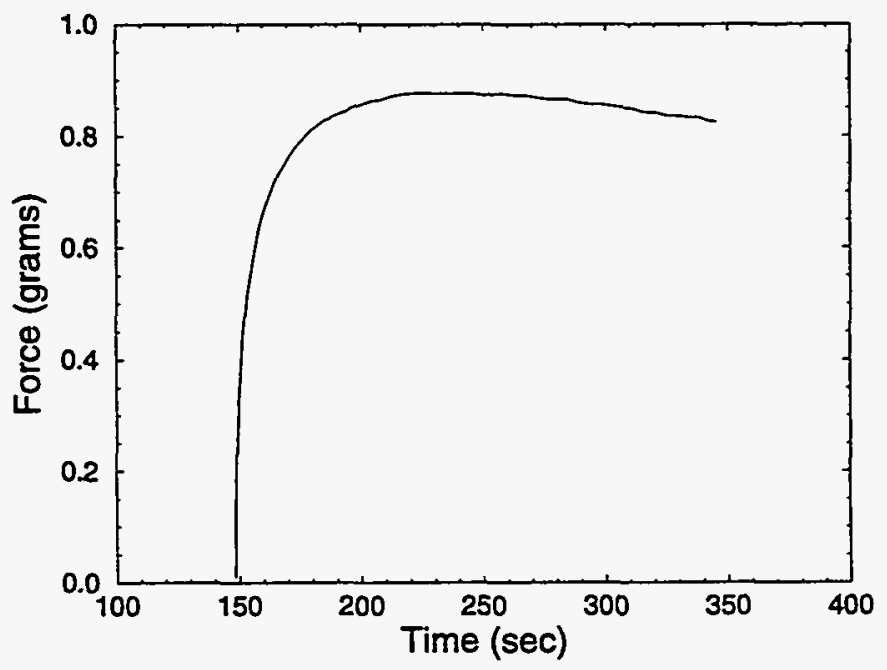

Figure 6. Force Measured in a Single Strand of SL 5149 Acrylate Resin With Constrained Ends Following a Second Laser Exposure

in the constrained strand following a second laser hit at about 150 seconds after the first draw. Note that the maximum force is attained in 60-90 seconds (after the second hit) after which stress relaxation begins. In tests conducted on the acrylate resin, cure-shrinkage forces were measured after subsequent hits, as well, indicating that the resin continues to react and shrink well after the first and second laser exposures.

To characterize the stiffness of a strand, stress relaxation tests were performed on samples created by 1,2, and 3 laser exposures (i.e., strands received 1,2, 3 laser hits). All tests were conducted by waiting a minimum of five minutes after the previous laser exposure before imposing any mechanical strain. During the stress relaxation tests, the electronic micrometer was programmed to impose a $0.5 \%$ axial strain on a one-inch strand over about 0.5 seconds. The decay in force was then measured as a function of time. Representative 


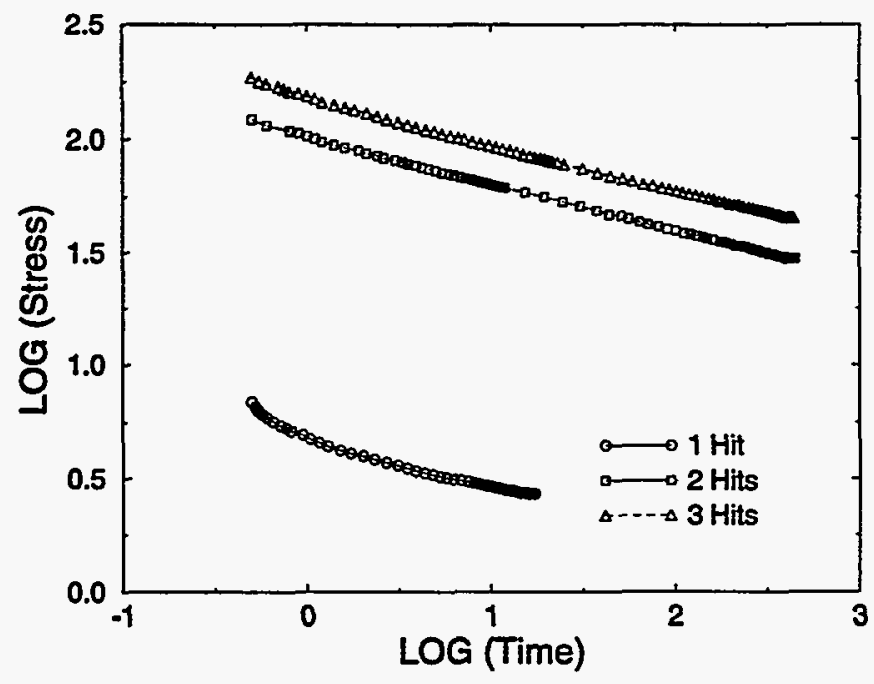

Figure 7. Stress Relaxation Measured in Single Strands of SL 5149 Acrylate Resin Following Multiple Laser Hits (Stress in psi and Time in seconds)

stress relaxation curves are plotted in Figure 7. Note that with each laser exposure the cross sectional area of the strand increases with the added depth of cure. Hence, the stress results plotted in Figure 7 reflect the fact that the measured forces have been normalized by three respective areas, 123.90E-6 in $^{2}$ for 1 laser hit, 146.73E-6 in $^{2}$ for 2 hits, and 153.73E-6 in ${ }^{2}$ for 3 hits. These correspond to the areas of the strand as measured following 1,2, and 3 laser exposures, respectively. From the stress relaxation data in Figure 7, an effective tensile stress relaxation modulus for the strand was obtained and fitted with an exponential series (i.e., Prony series). These data fits are shown in Figure 8 and depict the stress relaxation modulus as a function of the number of laser hits.

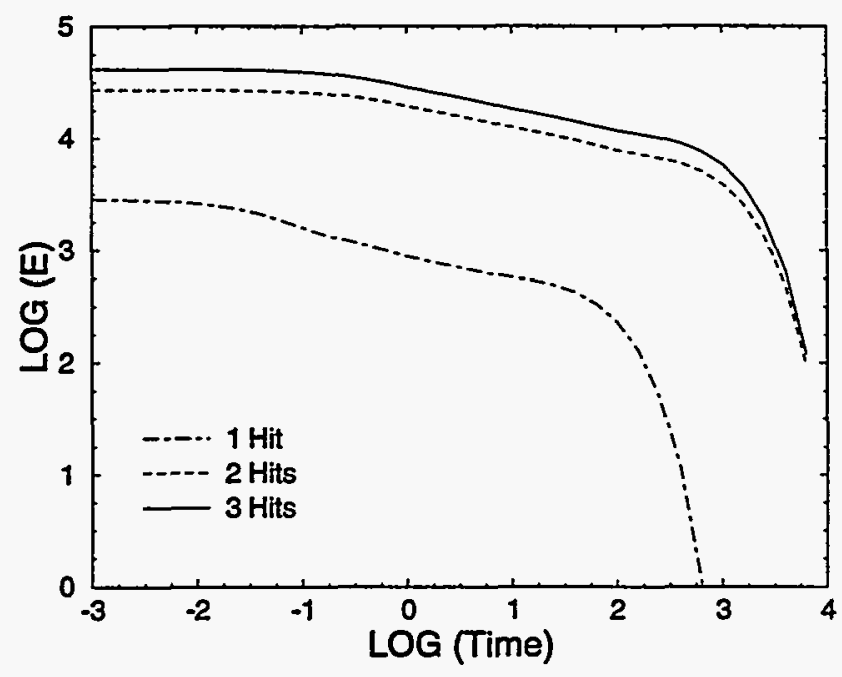

Figure 8. Prony Series Fits of Tensile Stress Relaxation Moduli for Single Strands of SL 5149 Acrylate Resin Following Multiple Laser Hits (Stress in psi and Time in seconds) 


\subsection{Solidification Modeling}

At the outset of the project, there was some hope that an elastic phenomenological model of solidification could be applied to the stereolithography process with sufficient resolution to qualitatively discern between build styles. Neglecting any exotherm and assuming isothermal conditions, the proposed elastic constitutive equation was of the form:

$$
\underline{\sigma}=\underset{\sim}{E}\left\{\underset{\varepsilon}{\varepsilon}-\underline{\varepsilon}_{c}\right\}
$$

where $\underset{\sigma}{\sigma}$ and $\underset{\sim}{\varepsilon}$ denote the stress and strain tensors, $\underset{\sim}{\mathrm{E}}$ is the elasticity operator and $\underset{\sim}{\varepsilon}$ is the cure shrinkage strain tensor. Soon after the stress relaxation tests had been initiated and simple artifact production had begun, it became apparent that time dependent material behavior was being observed. Hence, elasticity was abandoned and the following viscoelastic relation was pursued:

$$
\begin{gathered}
\sigma_{\mathrm{ij}}=2 \int_{0}^{t} G\{\xi(t)-\xi(\tau)\} \frac{d}{d t}\left\{\varepsilon_{i j}-\frac{\delta_{i j}}{3} \varepsilon_{k k}\right\} d \tau \\
+\delta_{i j} \int_{0}^{t} K\{\xi(t)-\xi(\tau)\} \frac{d}{d t}\left\{\varepsilon_{k k}-3 \varepsilon_{c}\right\} d \tau
\end{gathered}
$$

where indicial notation is employed and $\mathrm{G}$ and $\mathrm{K}$ are the shear and bulk stress relaxation functions, respectively, and $\xi$ is some intrinsic material time scale that depends on the degree of cure. Since only uniaxial tests were performed, the material functions were further simplified by assuming a constant Poisson's ratio and defining the bulk and shear moduli in terms of a uniaxial stress relaxation modulus, $\mathrm{E}(\mathrm{t})$ :

$$
\begin{aligned}
& G(t)=\frac{E(t)}{2(1+v)} \\
& K(t)=\frac{E(t)}{3(1-2 v)}
\end{aligned}
$$

The uniaxial stress relaxation function was assumed to be the same function as that plotted in Figure 7.

\subsection{Modeling Problems and Limitations}

After adopting the simplest viscoelastic constitutive equations, that might work (Equations 2-4), tests were planned and measured data were reviewed to determine whether a meaningful material characterization could be undertaken with the limited budget allocated for the project. All tests were evaluated within the context of the original intent of the project, namely 
to determine whether a phenomenological type material model could be used to analyze partbuilding. A review of existing data and capabilities revealed a host of problems:

1. In-situ cure-shrinkage data was only available for strands subjected to a single laser exposure. Because the density of the solidified strands exceeds that of the liquid resin, there is a tendency for the gelled strands to sink in the resin vat. The non-contact video method employed to measure cure shrinkage is not accurate if the tip of the strand is not clearly discernible. That is exactly the case as the strand sinks.

2. Although the Dayton experiments were able to measure the shrinkage during a second laser exposure, these experiments were conducted in a laboratory environment (not insitu) at different exposures from that employed in the SLA-250. Hence, there is some inconsistency between the data collected at Sandia and that collected under the Dayton contract.

3. The tensile stress relaxation tests were conducted on strands of resin that were not homogeneously cured. That is, there is a gradient in the degree of cure from top to bottom. Although this is known to create non-uniformity in actual shrinkage and stiffness, the strands were characterized in terms of their "average" properties. This approach was undertaken in an attempt to arrive at a simplified phenomenological material model.

4. The "average" stress relaxation function, $E(t)$, was measured as a discrete function of the number of laser exposures (Figure 8). The repeated laser hits took place after time intervals in which the reaction from one exposure had completed before the strand was subjected to an additional laser hit. In actual build styles, however, these conditions are not meet. Strands are repeatedly struck over shorter time intervals where the reaction from a prior hit may still be on-going when another laser strike takes place.

5. There was no data defining how to transition from one curve to another in Figure 8 . That is, how do you make the change from a single exposure "average" relaxation modulus to a double exposure "average" relaxation modulus? Because these are "average" properties in a nonhomogeneous material, one can consider a chemorheologically simple characterization analogous to a thermorheologically simple material. In the chemorheologically simple description, the shapes of the curve are the same but curves shift in the log time scale of Figure 8. A review of the data, however, shows the likely possibility of both vertical and horizontal shifts. There is no obvious or trivial way to distinguish how to separate these two shifts as a function of time during curing.

6. In an attempt to directly measure the cure shrinkage force in a constrained strand following the first laser exposure, the forces were too small to measure. Attempts to measure the force during subsequent exposures were successful. However, the force response was found to be a function of the time of exposure. That is, the magnitude and variation in the measured force depended on how many times the strand had been hit and when it was hit. This behavior cannot be characterized phenomenologically. Although the data gave some indication of the force generated during curing, it reflects the combination of shrinkage and stiffness in the strand without revealing the distribution of either function. 
7. The cross-sectional area of the strand changes with each laser exposure. The magnitude of the change may likewise vary with the number and time of exposures.

8. The actual reactions are known to be exothermic but no temperature affects were included or considered.

In reviewing this list of problems and limitations, it is clear that quantitative predictions require a much deeper understanding of the chemical kinetics and their affect on the viscoelastic material properties. A rigorous model would need to define:

1. the chemical kinetics including how the degree of cure varies in time and position following a specified laser exposure and how the exotherm and heat transfer problem materializes, and

2. the viscoelastic formalism which relates the viscoelastic material behavior (e.g., relaxation times, rubbery modulus, cure shrinkage, etc.) to the degree of cure and temperature.

This multi-disciplined problem would require a major research effort well beyond the scope of the current project. The current goal was to build the numerical platform (i.e., finite element architecture) that could be used to support model development and to determine what, if any, meaningful computations could be made with a phenomenological material model. The answer to this question appears to lie in limiting the problems to those for which representative phenomenological material properties are available. That is, problems where multiple laser exposures are spaced far enough apart in time for the moduli and shrinkage to be treated as isolated uncoupled functions of the number of laser hits. Even then, additional assumptions are required. An example problem is considered in the next section. 


\section{Demonstration Problems}

To demonstrate some of the generic capabilities of the finite element architecture that has been implemented to model the stereolithography process, two problems are presented. The first employs an elastic material model and is intended to exercise the element birthing procedure. The second problem makes use of the limited viscoelastic model to analyze two different build styles and compare the resulting curl predictions to the results measured on an artifact built with the SLA-250.

\subsection{Birthing Elastic Elements}

Using the finite element mesh in Figure 2, the process build for a simple cantilever beam has been analyzed using a totally elastic material model. The modulus of elasticity and Poisson's ratio for the solidified material were assumed to be equal to 150,000 psi and 0.30 , respectively. Since this problem was intended to be no more than a demonstration of the finite element birthing methodology, the time of solidification was neglected and an instantaneous cure-shrinkage of $0.2 \%$ was imposed as each element was activated. No time delay was included after processing a layer. The depth of cure was assumed to correspond to the 0.010inch layer thickness, and each element was hit by the laser only once. The finite element mesh block consists of six 0.010 inch-thick layers stacked vertically in the Z-direction and contains 1560 nodes and 1044 elements. The length of the mesh block is 0.29 inches and the width is 0.03 inches. From this block of finite elements, the bottom five layers were processed to build a cantilever beam according to the geometry shown in Figure 9. To define the build process,

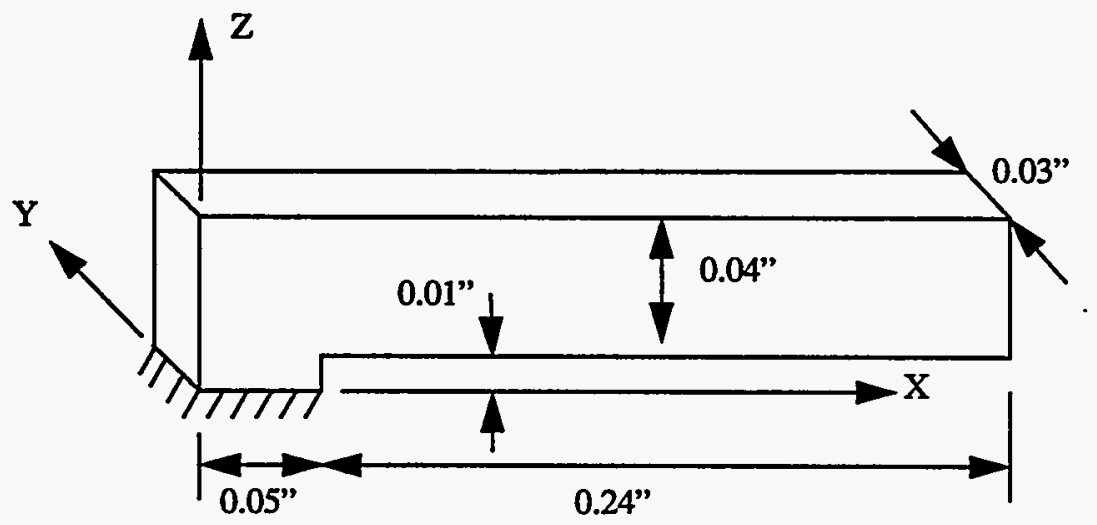

Figure 9. Geometry of Cantilever Beam Analyzed During Process Build

two distinctly different layer histories were chosen. A schematic of the laser paths and the numbered sequence of vectors drawn in each layer is shown in Figure 10. In Scheme I, the laser paths were constructed using parallel vectors running along the length of the beam. After drawing the base as the first layer, subsequent beam layers were drawn with three vectors, the first one passing through the plane of the centerline of the beam and the second and third positioned on opposite sides of the first vector. Scheme II was fundamentally different in two ways: 1) the vectors were drawn in a direction transverse to the axis of the beam, and 2) the vectors along the cantilever were laid down in two passes. In the first pass, the vectors were placed 0.020 inches apart leaving a 0.010 inch gap between strands. These gaps then were filled on a return pass back down the length of the beam. 

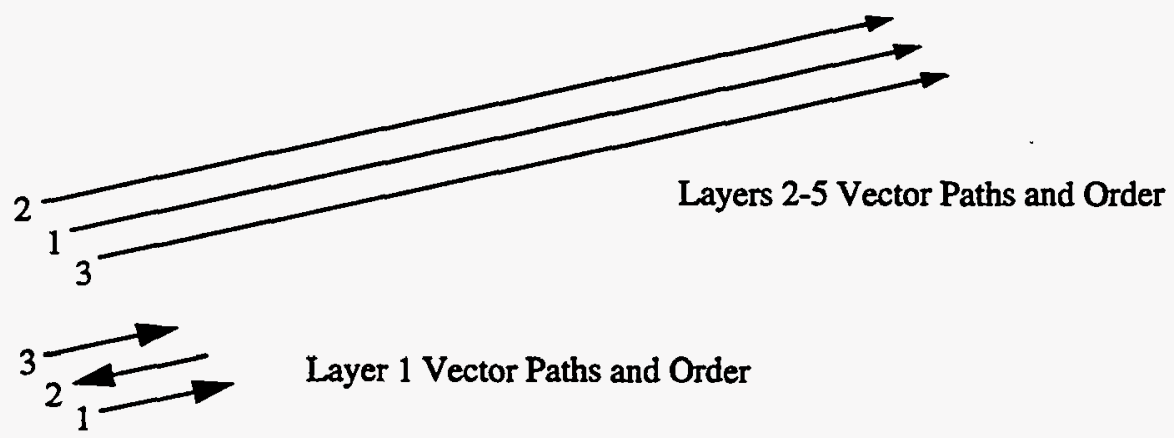

a) Build Style I
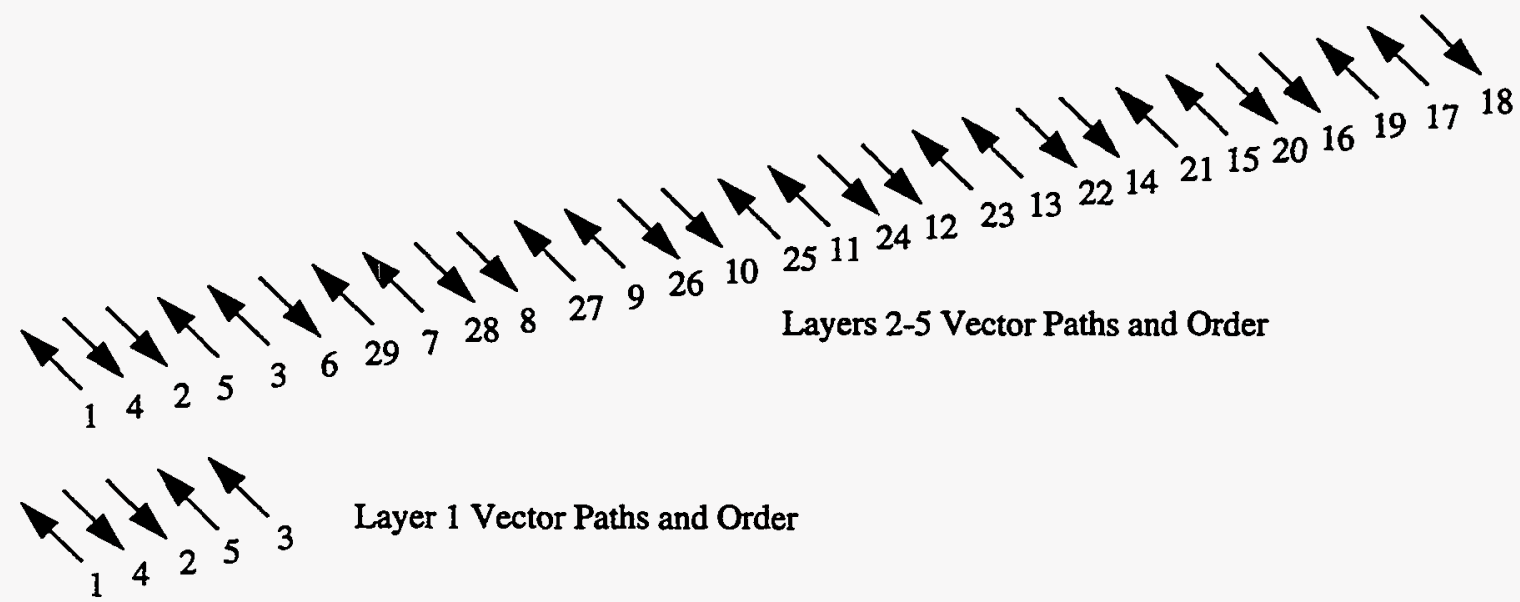

Layer 1 Vector Paths and Order

b) Build Style II

Figure 10. Schematics Illustrating the Vector Paths and Drawing Sequence for the Two Build Styles Used in the Analysis of the Cantilever Beam

Plots of deformed meshes obtained from the analysis of the Scheme I build style are shown in Figure 11. One mesh depicts the total beam, and the other is an expanded view of a section taken from the end of the beam. The plotted deformations are to scale. An examination of the deformed elements shows that the bottom surface of the cantilever beam (i.e., second mesh layer from bottom of the block shown in Figure 2) is prone to a significant amount of curl. When the fluid level of the next layer (i.e., third mesh layer from the bottom) is added to the problem, there is a definite nonuniformity in the layer thickness proceeding from the fixed end to the tip of the cantilever. This is apparent in the "squashed" appearance of the bottom row of elements making up that layer. The added fluid does tend to level-out the curl on the top surfaces of the beam layers but the underside of the cantilever continues to curl with the addition of layers. The amount of curl induced by these extra layers does, however, decrease due to the growing moment of inertia of the beam cross section. The Z-displacement along the bottom face of the cantilever beam (layer 2 in the finite element mesh block) has been plotted as a function of the distance measured from the fixed end $(X=0)$ to the beam tip $(X=0.29)$ in 

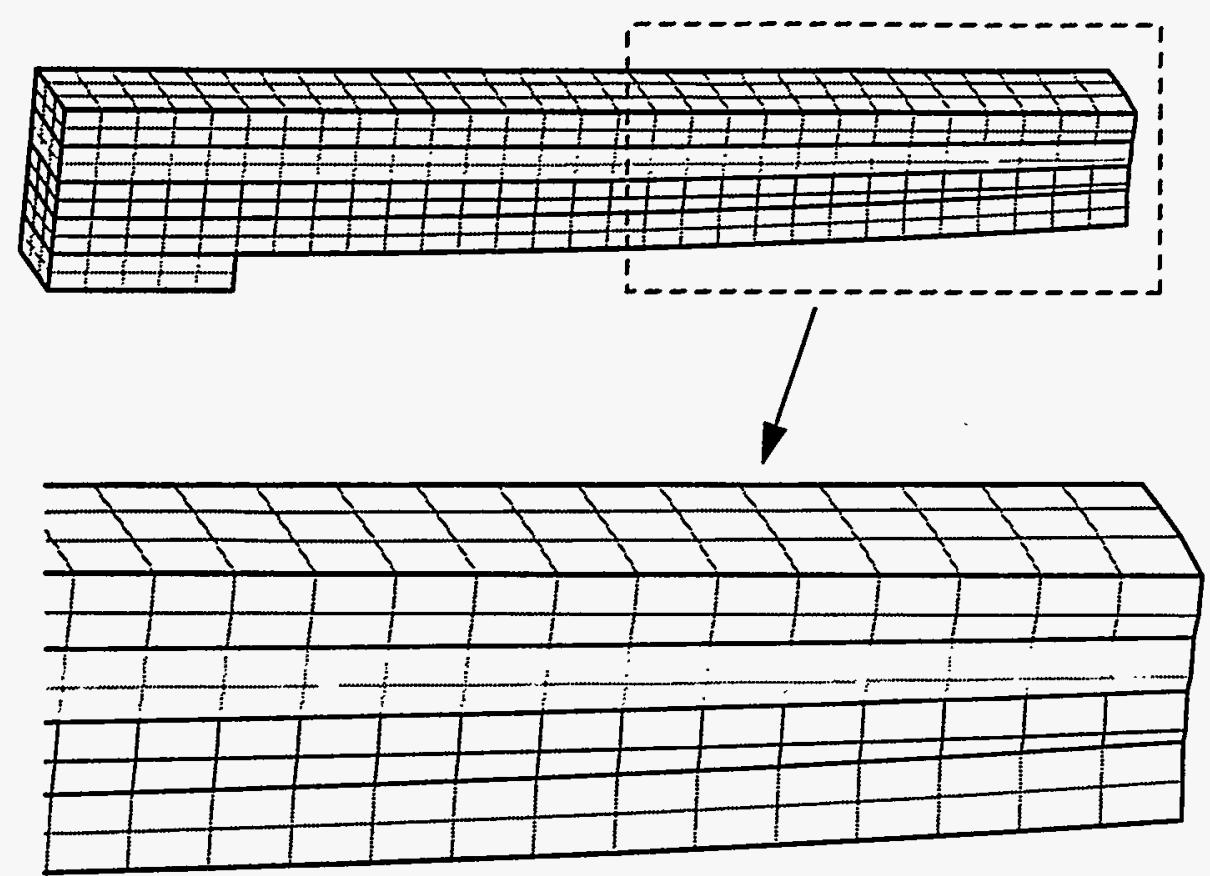

Figure 11. Plots of the Deformed Mesh Obtained from the Analysis of the Cantilever Beam Built According to the Scheme I Laser History

Figure 12 and Figure 13 for the Scheme I and Scheme II build styles, respectively. Each figure contains four curves corresponding to the deformation present after each of the four layers making up the beam cross section have solidified. The magnitude of the curl resulting from the Scheme I build style is about 5 times greater than the curl produced by the Scheme II build. The difference stems from the order of solidification. In Scheme I, solidification and shrinkage are staged along the axis of the cantilever starting at the fixed end and moving to the free end of the beam. In this case, the curl produced by element birth is additive. In Scheme II, strands are drawn across the transverse section of the beam leaving gaps between strands allowing much of the shrinkage to be absorbed locally without curling the length of the beam. The fact that these analyses assumed an elastic material response with an instantaneous shrinkage does tend to magnify the amount of curl. However, these analyses do emphasize the way that build history can affect the curl in a part.

\subsection{Viscoelastic Analysis of Contrived Build Styles}

The second demonstration problem employed a crude viscoelastic representation of the solidification incurred in a contrived build style using the acrylate resin. The restrictions in the model were dictated by the conditions under which the phenomenological viscoelastic material data were collected. Namely, properties were obtained following the reaction initiated by uncoupled laser exposures on resin strands drawn in the SLA-250. Data from repeated laser exposures were taken only after allowing sufficient time for the reaction due to a prior laser exposure to come to completion. Hence, there was no coupling between the reactions initiated by multiple laser hits. This limitation was necessary to accommodate the phenomenological 


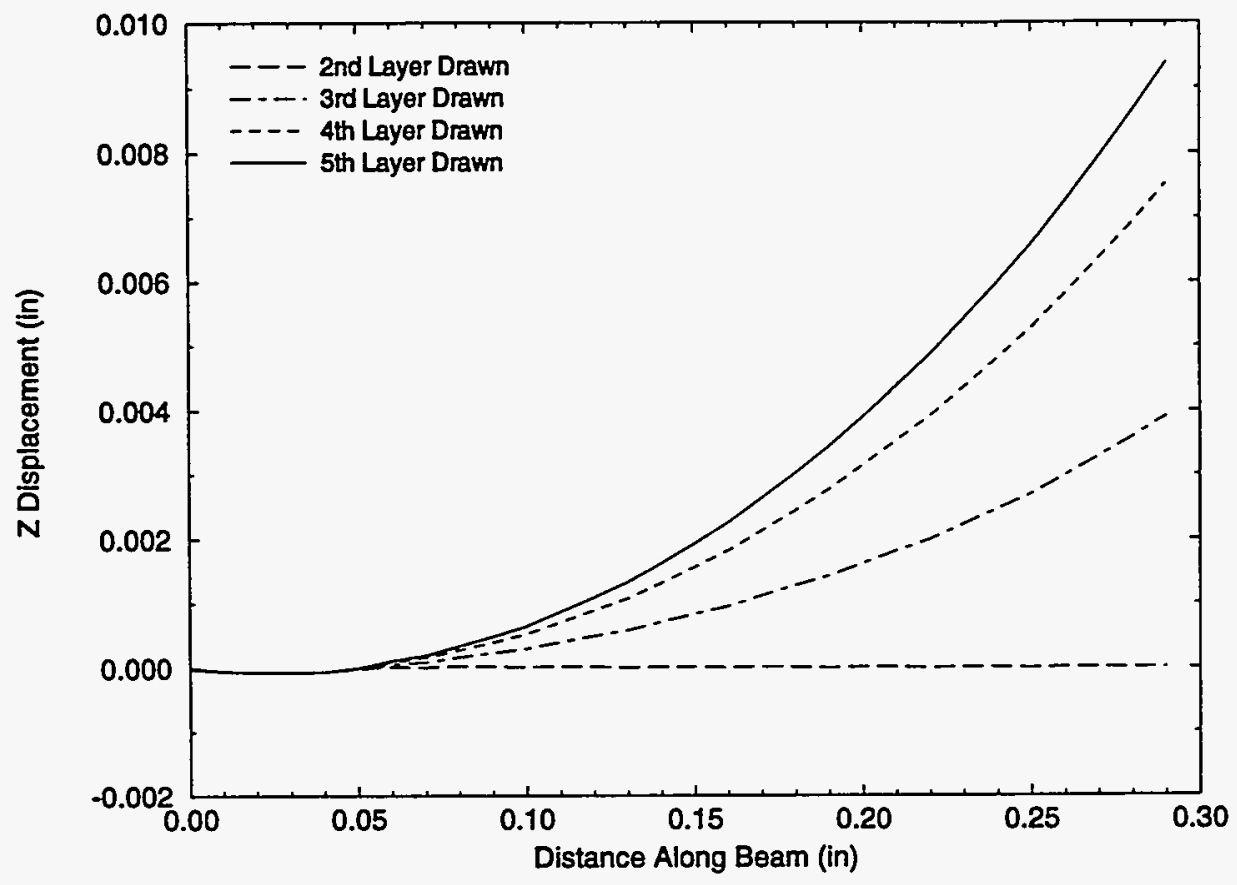

Figure 12. Plots of Z-Displacement Along the Bottom Face of the Cantilever Beam Following the Solidification of 4 Layers Drawn Using Scheme I

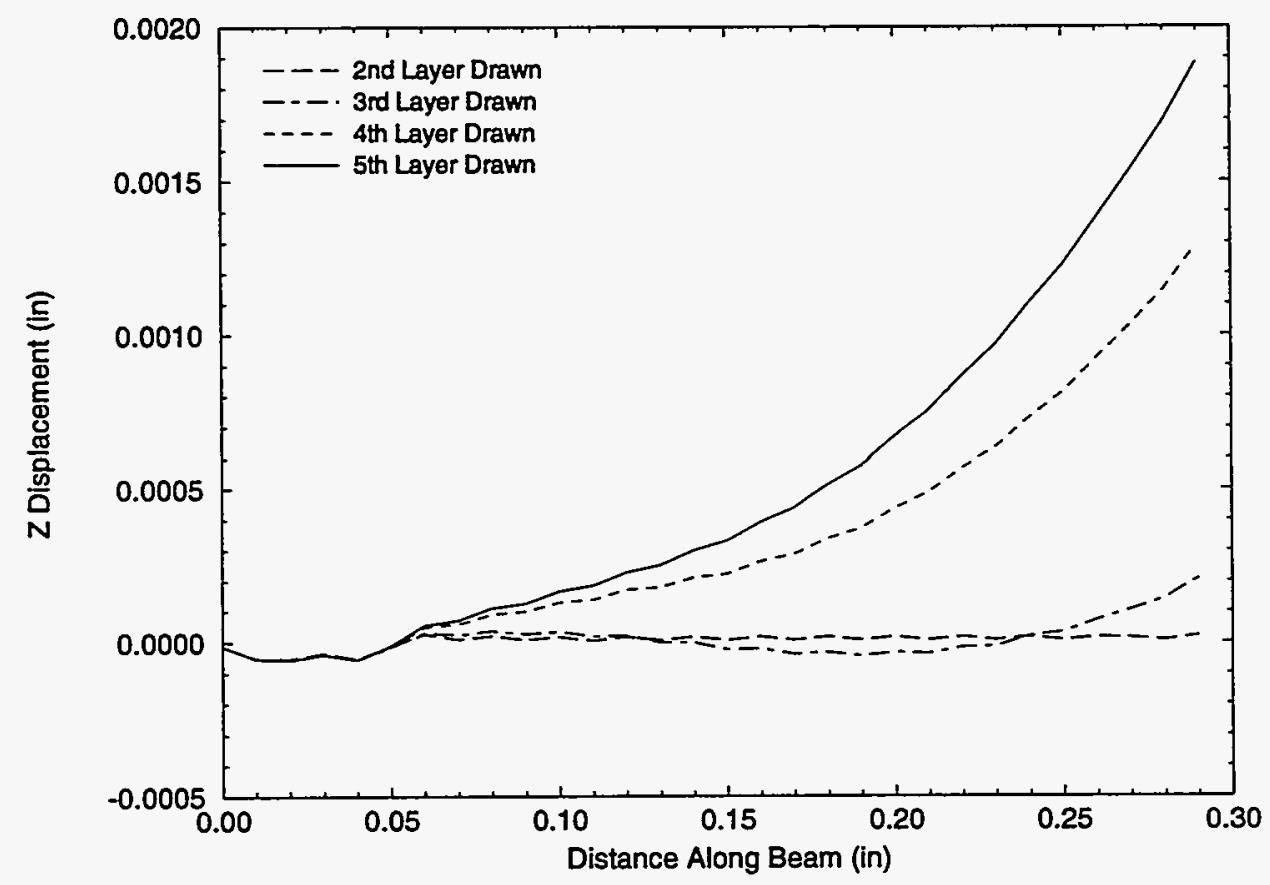

Figure 13. Plots of Z-Displacement Along the Bottom Face of the Cantilever Beam Following the Solidification of 4 Layers Drawn Using Scheme II 
material model which lacks any formalism for modeling the behavior found in coupled reactions. To make use of the phenomenological material representation in modeling the stereolithography process, artifacts must be built under similar limitations. That is, strands must be drawn under one exposure and allowed to fully react before being subjected to a subsequent exposure. Using special software provided by the University of Dayton's Rapid Prototyping Development Laboratory such a build style was created to construct two cantilever beams similar to those described in the previous demonstration problem.

The two beams built and modeled for this demonstration mimicked those described in the last example with the exceptions that each strand in each layer received two laser exposures, and the beam was slightly smaller. The beam geometry used in the build is shown in Figure 14. During the build, the second exposure occurred only after allowing time for the

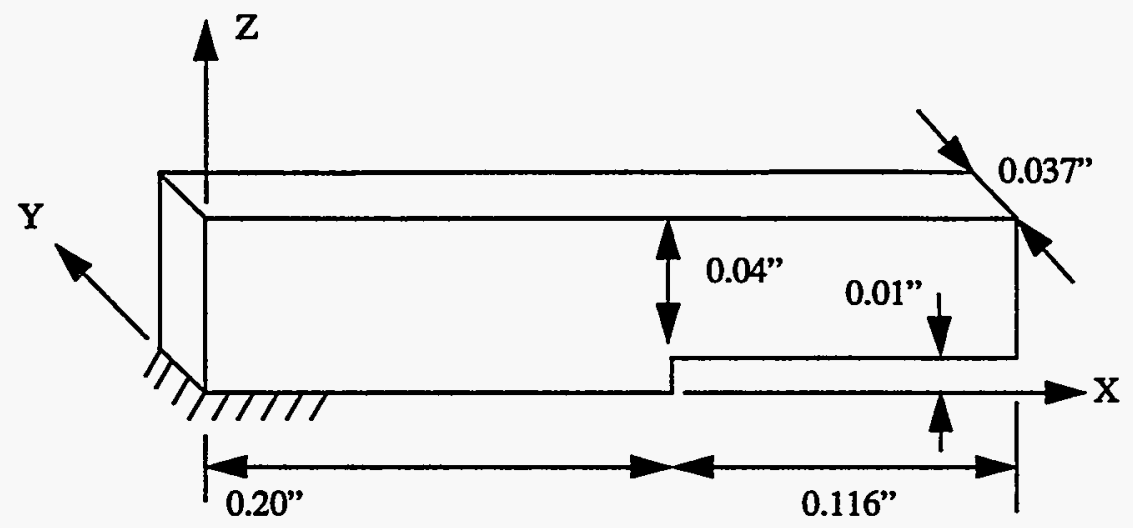

Figure 14. Cantilever Beam Geometry Used in Artifact Build and Modeling for Demonstration Problem 2

reaction from the first exposure to come to completion. Under the Scheme I longitudinal build (see Figure 10a), vector paths 1, 2, and 3 were drawn (exposure 1) followed by a 200 second delay and then a second laser exposure. The programmed delay between build layers during the elevator dip and squeegeeing operation was likewise about 200 seconds. In the transverse build style of Scheme II, the procedure to doubly expose each layer was more complicated. The base and first layer of the overhang in the cantilever were drawn using longitudinal vectors as in Scheme I to provide a support for the transverse vectors in the remaining layers. Without a lower layer for attachment, these short vectors would drift away in the resin vat before they could be struck a second time. The procedure for constructing the transverse vectors in the subsequent layers is described as follows using the 5 vectors in Figure 15:

1. Expose transverse vectors 1,2 , and 3 and then wait about 200 seconds for these vectors to react (solidifying and shrinking)

2. Fill in the gaps between these vectors by drawing vectors 4 and 5 and then wait another 200 seconds for these vector strands to cure

3. Expose vectors 1,2, and 3 for a second time and then wait another 200 seconds

4. Expose the in-between vectors, 4 and 5, and then wait 200 seconds 
5. Move on to next layer repeating the process after elevator dip and squeegeeing

In the actual beam, substantially more vectors were required to achieve the necessary length. However, the procedure for exposing alternate transverse vectors, waiting for the reaction to terminate, and then exposing the in-between vectors remains the same.

To model the two build schemes, data is needed on the resin behavior following two time-separated laser exposures. Some of that data is available from the testing, but some had to be approximated. That is why these results should be viewed as demonstration problems and not quantitative predictions. The acrylate shrinkage strain from a single laser strike is plotted in Figure 6. However, there is no data available on the corresponding evolution in relaxation modulus. All that is known is that a constrained strand generates less than 0.02 grams of force following a single laser exposure (ref. Section 3.2). To model a similar behavior following the first laser hit, the resin was assigned the relaxation modulus depicted by the 1-hit curve in Figure 8, and the reduced time function in Equation (2) was adjusted to allow relaxations to occur fast enough for the cure-shrinkage force to remain below 0.02 grams during cure. In this way, the stiffness of the strand is assumed to be similar to what was measured experimentally. This was accomplished by using a shift factor, $\Phi$, that varied from 5000 to 1 over a 180 second time frame following the first laser exposure:

$$
\begin{gathered}
\xi(t)=\int_{t_{\text {hit }}}^{t} \Phi(s) d s \\
\Phi(t)=\operatorname{Max}\left(5000\left(1-\frac{t-t_{\text {hit }}}{180}\right), 1\right) \text { for } t_{\text {hit }} \leq t \leq 180 \text { seconds }
\end{gathered}
$$

After 180 seconds, the shift factor is reduced to a value of one, yielding the apparent modulus of the one-hit curve in Figure 8. The force measured in a constrained strand after a second laser exposure was used to back out an approximate curing stress relaxation function for the second exposure. This was done by noting that according to the data in Figure 8, an approximate vertical shift can be used to get from one relaxation modulus to another. Through numerical experiments, it was determined that when the glassy modulus is scaled from 1300 psi (i.e., the 1 hit curve) to 27170 psi (i.e., the 2 hit curve) over the 50 second period immediately following the second laser exposure, the Dayton 2nd hit cure-shrinkage strains are reasonably approximated. The data and model approximation are plotted in Figure 16. The 1300 psi and

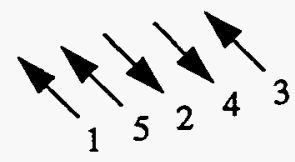

Figure 15. Transverse Vectors Used to Illustrate Scheme II Build Scheme 


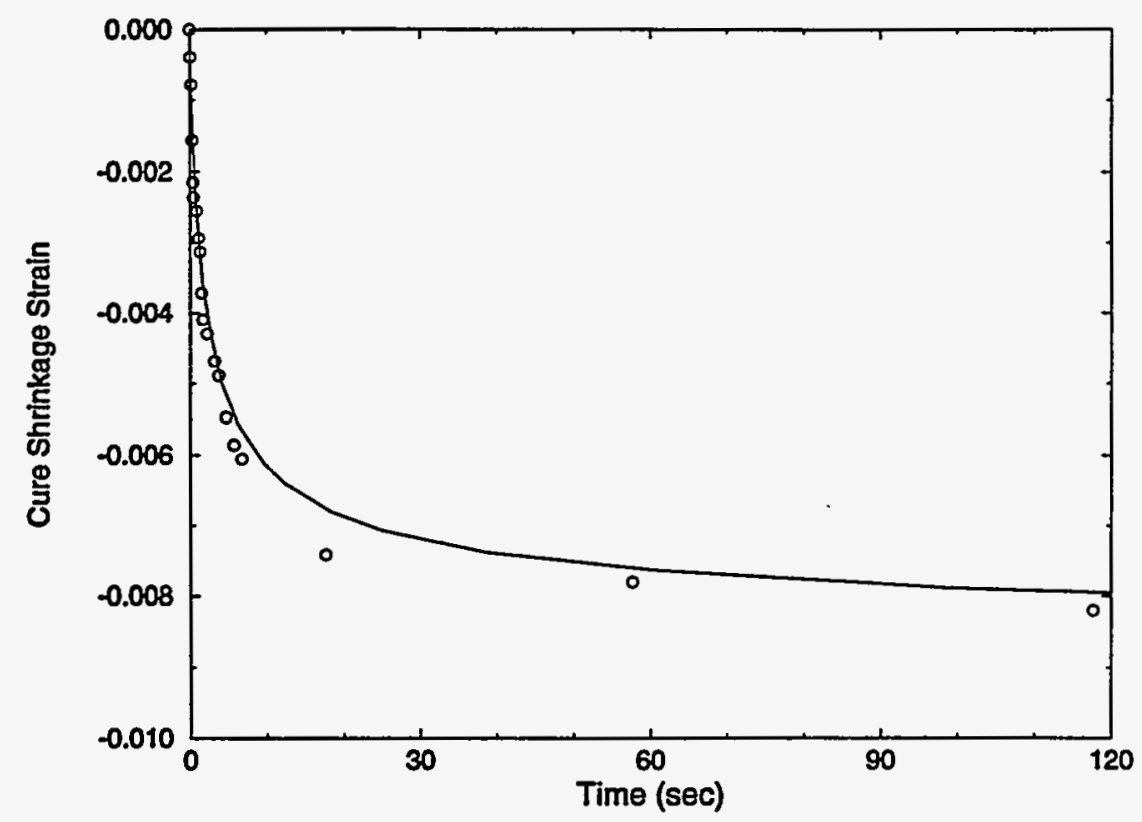

Figure 16. Dayton 2nd Laser Exposure Data Compared to Model Approximation

27170 psi values are taken directly from the glassy moduli fitted to the single and double exposure curves in Figure 8.

Based on the material data and assumptions described in the previous paragraphs, the cantilever beam was analyzed for both the Scheme I and Scheme II build styles. Figure 17 contains plots of the curl (Z-displacement) on the lower surface of the cantilever measured as

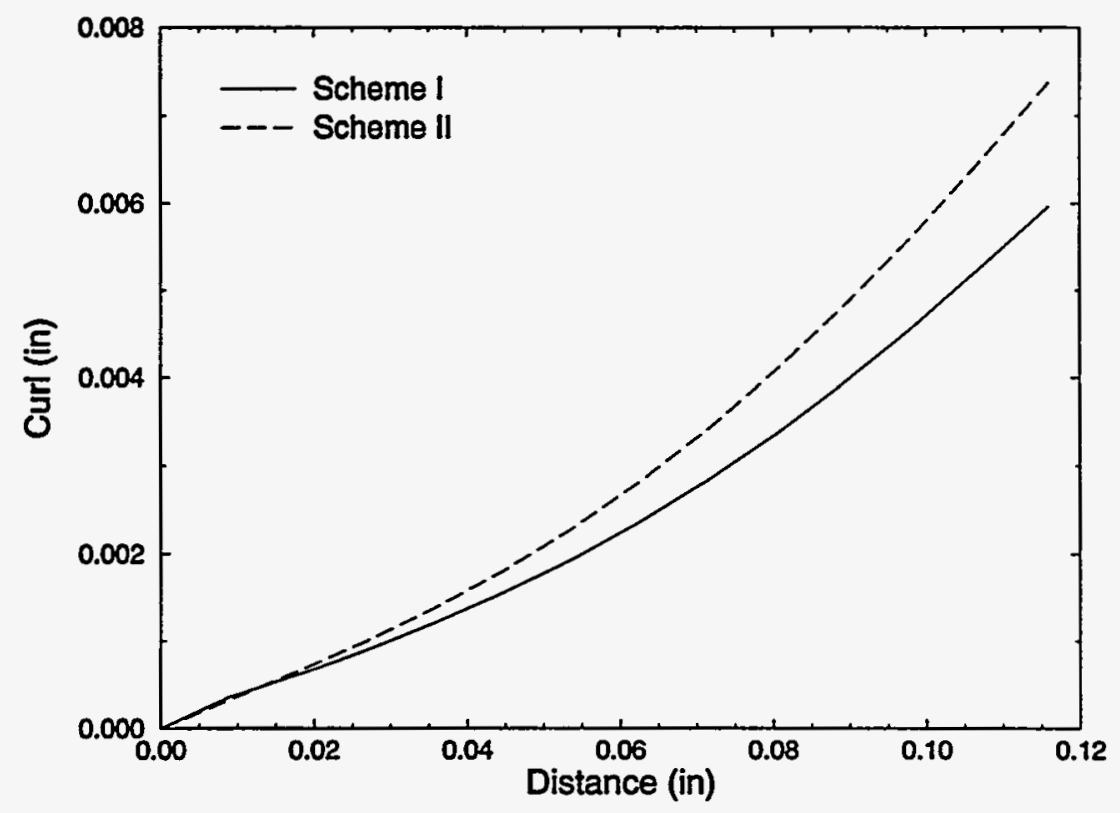

Figure 17. Comparison Between the Finite Element Curl Predictions Obtained by Modeling the Scheme I and Scheme II Build Styles in Cantilever Beam 
a function of the distance from the base of the cantilever. This reflects the deformation predicted at the completion of the build. When these predictions are compared to the results obtained in the first demonstration problem (see Figure 12 and Figure 13), a major difference is apparent. In the first demonstration problem, the transverse build style of Scheme II was found to produce beam curling that is 5 times less than that generated under the longitudinal build style of Scheme I. However, in the current problem, the transverse build style produces slightly more curl than the longitudinal build. This difference is attributed to the incorporation of time dependent shrinkage. When the shrinkage occurs instantaneously, as in the first demonstration problem, the magnitude of the curl is determined by the elastic properties of the resin and the stiffness of the elements activated at the time the shrinkage takes place. This shrinkage occurs one element at a time, sequentially, according to the order of the Figure 10 build vectors. In contrast, the shrinkage encountered in the second demonstration problem transpires over 2-3 minutes while the time required to expose a layer is actually a fraction of a second. This means that the layer shrinkage occurs as if all exposed elements were exposed at nominally the same time. When the gaps between existing vectors are filled (e.g., vectors 4 and 5 in Figure 15 are being drawn), the shrinkage is constrained not only by the underlying material from previously cured layers but from the adjacent vectors (i.e., vectors 1,2 , and 3 ) which have already solidified. The added in-plane constraint and near concurrent shrinkage of the gap-filling vectors, translates into a larger beam curl than is present if the gap-filling vectors cure instantaneously in the sequence in which they were drawn.

The curl deformations were measured in cantilever beams that were built in the SLA-250 according to the Scheme I and Scheme II build styles. These results are plotted in Figure 18. Note that the data have been zeroed at some point nominally located about 0.02 inches from the base of the cantilever. This action was necessary to provide a well defined reference point

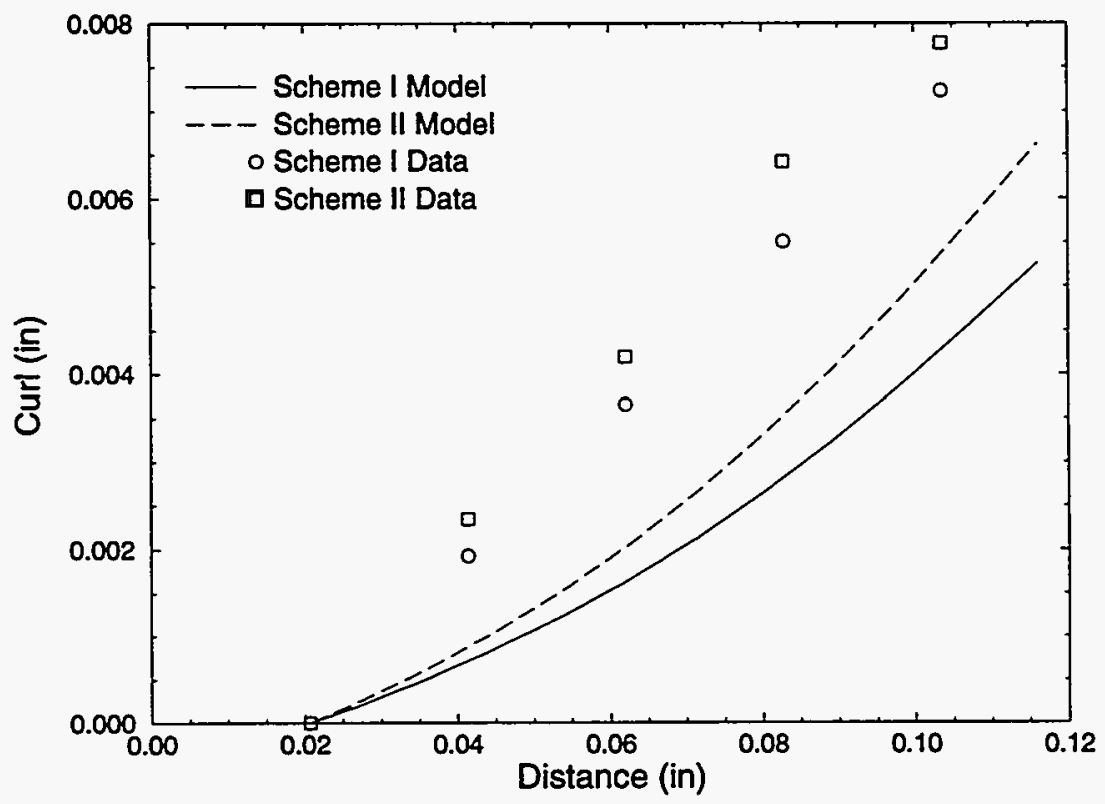

Figure 18. Curl Measured and Predicted in Cantilever Beams Built in SLA-250 According to the Scheme I and Scheme II Build Styles 
on the lower surface of the beam from which to make relative curl measurements. Although the finite element mesh has a $90^{\circ}$ corner providing a well defined origin for the abscissa in the plot, the actual parts have rounded corners which create some uncertainty in the position. For clarity of comparison, the finite element model predictions likewise have been modified to comply with this relative shift and plotted along with the measured data in Figure 18. Both the model predictions and the measurements indicate that the transverse build style in Scheme II yields slightly greater amounts of curl than the longitudinal build style. Differences between the magnitudes of the curl predicted and measured are understandable given the nature of the material approximations and modeling assumptions employed in the analysis. In particular, the overcure created by the laser exposure actively penetrating lower layers has been completely ignored, and the formalism by which the relaxation modulus evolves is unknown. Nevertheless, the model problem does serve as a suitable demonstration of the use of the time dependent material behavior in a phenomenological material model, and it is able to capture qualitatively the observed difference in response between the Scheme I and Scheme II build styles. 


\section{Conclusions}

A capability for performing finite element analyses of the stereolithography process has been developed. A methodology and code architecture have been structured to allow finite elements to be birthed (activated) according to a prescribed order mimicking the procedure by which a laser is used to cure and build-up surface layers of resin to construct a three dimensional geometry. In its present form, the finite element code incorporates a severely restrictive phenomenological material model of solidification that is based on the shrinkage and relaxation observed following isolated, uncoupled exposures of the photocurable resin to a laser. Although the phenomenological material model has been used in a simple demonstration problem to qualitatively make a distinction between two build styles, these builds were designed so that the build vectors, once exposed to the laser, could react and have time to finish reacting before being subjected to a second laser strike. This is an unrealistic assumption that prohibits use of the model in realistic builds. To continue development of the methodology, the chemical kinetics and thermodynamics of the reaction and the formalism relating the kinetics to the viscoelastic behavior of the resin must be understood. 


\section{References}

[1] SLA-250 User Guide, 3D Systems, Inc., April 1991.

[2] Marutani, Y. and Nakai, T., Laser Research 17, 410 (1989).

[3] Flach, L. and R. P. Chartoff, "Laser Scan Rates and Shrinkage in Stereolithography," Proceedings of the Fourth International Conference on Rapid Prototyping, University of Dayton, Dayton, Ohio, June 14-17, 1993.

[4] Horton, L., Gargiulo, E., and M. Keefe, "An Experimental Study of the Parameters Affecting Curl in Parts Created Using Stereolithography,"'Proceedings of the Solid Freeform Fabrication Symposium, The University of Texas at Austin, Austin, Texas, August 9-11, 1993.

[5] Jacobs, P. F., Rapid Prototyping and Manufacturing - Fundamentals of Stereolithography, Society of Manufacturing Engineers, 1992.

[6] Pang, T.H., "Stereolithography Epoxy Resin Development: Accuracy and Dimensional Stability," Proceedings of the Solid Freeform Fabrication Symposium, The University of Texas at Austin, Austin, Texas, August 9-11, 1993.

[7] Blanford, M. L., "Release 1.1 of JAS3D,", Memo to Distribution, 7/20/95, Sandia National Laboratories, Albuquerque, NM.

[8] Guess, T. R., Chambers, R. S., Hinnerichs, T. D., "Epoxy and Acrylate Stereolithography Resins: In-Situ Property Measurements," Sandia National Laboratories, Albuquerque, NM, SAND95-2871, January, 1996.

[9] Chambers, R. S., Guess, T. R., and Hinnerichs, T. D., "A Phenomenological Finite Element Model of Part Building in the Stereolithography Process," Proceedings of the Sixth International Conference on Rapid Prototyping, University of Dayton, Dayton, Ohio, June 4-7, 1995.

[10] Guess, T. R., Chambers, R. S., Hinnerichs, T. D., McCarty, G. D., and R. N. Shagam, "Epoxy and Acrylate Stereolithography Resins: In-Situ Measurements of Cure Shrinkage and Stress Relaxation," Proceedings of the Sixth International Conference on Rapid Prototyping, University of Dayton, Dayton, Ohio, June 4-7, 1995.

[11] Guess, T. R. and Chambers, R. S., "In-Situ Property Measurements on Laser-Drawn Strands of SL 5170 Epoxy and SL 5149 Acrylate," Proceedings of the Sixth Solid Freeform Fabrication Symposium," Austin, Texas, August, 1995.

[12] Weissman, P. T., Chartoff, R. P., Rodfigues, S. J., and S. Linden, "Real Time Measurements of Linear Shrinkage During Laser Photopolymerization: Implications Concerning Post Cure Shrinkage," Proceedings of the Fourth International Conference on Rapid Prototyping, University of Dayton, Dayton, Ohio, June 14-17, 1993.

[13] Contract with Dr. Richard P. Chartoff, The University of Dayton Research Institute, Materials Engineering Division, Dayton, Ohio, PR No. AI-7340, 12/15/93. 


\section{Distribution:}

3 University of Dayton

Rapid Prototyping Development Laboratory

Dayton, Ohio 45469-0130

Attn.: Dr. Richard P. Chartoff

Dr. Allan J. Lightman

Dr. Jill S. Ullett

2 3D Systems

26081 Avenue Hall

Valencia, CA 91355

Attn.: Mr. Charles W. Hull

Dr. Paul F. Jacobs

1 Dr. Manfred Hofmann

Ciba-Geigy AG

FZM-185.003

P. 0.64

CH-1723 Marly-1

Switzerland

$\begin{array}{llll}1 & \text { MS-0960 } & 1400 & \text { J. Q. Searcy } \\ 1 & \text { MS-0961 } & 1403 & \text { J. A. Sayre } \\ 1 & \text { MS-0958 } & 1472 & \text { J. Ledman } \\ 1 & \text { MS-0958 } & 1471 & \text { G. D. McCarty } \\ 1 & \text { MS-0958 } & 1472 & \text { J. A. Emerson } \\ 5 & \text { MS-0958 } & 1472 & \text { T. R. Guess } \\ 1 & \text { MS-0958 } & 1472 & \text { M. E. Stavig } \\ 8 & \text { MS-0958 } & 1484 & \text { C. L. Atwood } \\ 1 & \text { MS-0958 } & 1484 & \text { M. L. Griffith } \\ 1 & \text { MS-0958 } & 1484 & \text { L. D. Harwell } \\ 1 & \text { MS-0958 } & 1484 & \text { P. G. Stromberg } \\ 1 & \text { MS-0958 } & 1484 & \text { B. T. Pardo } \\ 1 & \text { MS-0958 } & 1484 & \text { D. E. Reckaway } \\ 1 & \text { MS-0328 } & 2674 & \text { R. N. Shagam } \\ 1 & \text { MS-9403 } & 8230 & \text { J. Spingarn } \\ 1 & \text { MS-1436 } & 4503 & \text { LDRD Office } \\ 1 & \text { MS-0841 } & 9100 & \text { P. J. Hommert } \\ 1 & \text { MS-0835 } & 9102 & \text { R. D. Skocypec (Route to 9111) } \\ 1 & \text { MS-0833 } & 9103 & \text { J. H. Biffle (Route to 9116) } \\ 1 & \text { MS-0828 } & 9104 & \text { E. D. Gorham (Route to 9114, 9115) }\end{array}$


1 MS-0834 9112 A. C. Ratzel (Route to 9113)

1 MS-0443 9117 H.S. Morgan

1 MS-0437 9118 E.P. Chen (Acting)

1 MS-0443 QA File

10 MS-0443 9117 R. S. Chambers

1 MS-0439 $9234 \quad$ D. R. Martinez

1 MS-0439 9234 T.D. Hinnerichs

1 MS-0439 $9234 \quad$ M. S. Eldred

1 MS-0439 $9234 \quad$ W. R. Witkowski

1 MS-0367 $1815 \quad$ D. B. Adolf

5 MS-0899 4414 Technical Library

2 MS-0100 7613-2 Document Processing for DOE/OSTI

1 MS-9018 8523-2 Central Technical Files

1 MS-0619 12615 Print Media 


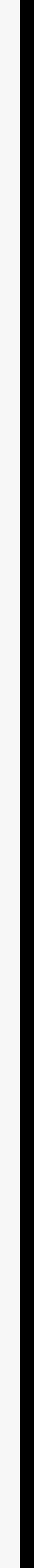

